\title{
De novo metastatic breast cancer: Subgroup analysis of molecular subtypes and prognosis
}

\author{
LI ZHANG $^{1 *}$, ZHIJUN LI $^{1 *}$, JIE ZHANG $^{1}$, YANSHENG WU ${ }^{2}$, YUYING ZHU ${ }^{1}$ and ZHONGSHENG TONG ${ }^{1}$ \\ ${ }^{1}$ Department of Breast Oncology, Key Laboratory of Breast Cancer Prevention and Therapy, \\ National Clinical Research Center for Cancer, Tianjin Medical University Cancer Institute and Hospital; \\ ${ }^{2}$ Department of Maxillofacial and Otorhinolaryngology, Head and Neck Surgery, National Clinical Research Center for Cancer, \\ Tianjin Medical University Cancer Institute and Hospital, Tianjin 300060, P.R. China
}

Received April 13, 2019; Accepted December 20, 2019

DOI: $10.3892 / 01.2020 .11359$

\begin{abstract}
The purpose of the present study was to analyze the clinical and pathological characteristics, treatment, and prognosis of de novo metastatic breast cancer (DnMBC). Information regarding 1,890 patients treated for advanced breast cancer at the Tianjin Medical University Cancer Hospital between January 2008 to December 2017 was collected. Clinicopathological characteristics, treatments and outcomes of these patients were compared using the chi-square test, log-rank test, and Cox regression analysis. A total of 171 patients were diagnosed with DnMBC. The median age at diagnosis was 53 years (range, 23-77). The percentage of T4 staging was higher $(37.4 \%), 69.6 \%$ of patients were estrogen receptor (ER) positive, $59.1 \%$ were progesterone receptor positive, $29.8 \%$ had positive human epidermal growth factor receptor 2 (HER2) status, $68.4 \%$ had $\mathrm{Ki}-67 \geq 20 \%, 55 \%$ had oligometastasis at the initial diagnosis, $\sim 87.7 \%$ were treated with chemotherapy initially and $24 \%$ received palliative surgery for the primary tumor. After a median follow-up time of 26 months, the median progression-free survival (PFS) and overall survival (OS) among patients with DnMBC were 11 (8.7-13.3) months and 34 (27.9-40.1) months, respectively. In the multivariable model, ER status and sites of first metastasis (oligometastasis or polymetastasis) were identified to be independent predictors of PFS $(\mathrm{P}<0.05)$; ER status, primary tumor stage, and surgical treatment of primary tumors were identified to be independent predictors of $\mathrm{OS}(\mathrm{P}<0.05)$. In conclusion,
\end{abstract}

Correspondence to: Dr Zhongsheng Tong, Department of Breast Oncology, Key Laboratory of Breast Cancer Prevention and Therapy, National Clinical Research Center for Cancer, Tianjin Medical University Cancer Institute and Hospital, 1 West Huanhu Road, Tianjin 300060, P.R. China

E-mail: tongzs163@163.com

*Contributed equally

Key words: breast cancer, de novo metastatic, clinicopathological characteristics, prognosis, subtypes, primary tumor surgery the clinicopathological characteristics of DnMBC are greater invasiveness and a higher risk of progression. Palliative surgical treatment may improve the prognosis of HR+/HER2-patients with oligometastasis. Therefore, individualized treatment as required is particularly important.

\section{Introduction}

Breast cancer is one of the most common malignant tumors in women. In China, the incidence of breast cancer ranks first and its mortality rate ranks second after that of lung cancer (1). Clinical studies have demonstrated that $\sim 20-30 \%$ of early breast cancer will recur and metastasize to become advanced breast cancer, that is, recurrent metastatic breast cancer, while $\sim 3-10 \%$ of breast cancer patients have distant metastasis at the initial diagnosis, namely de novo metastatic breast cancer (DnMBC) (2-4). Patients with DnMBC often lose the opportunity of radical surgery when they were diagnosed for the first time and their psychological status and quality of life are seriously affected. At the same time, because of its special clinicopathological characteristics and since its proportion in advanced breast cancer patients is increasing year by year, more and more clinicians are paying attention to it (5). Prior reports state that the clinicopathological features of DnMBC were greater invasiveness. The proportion of multiple metastasis and visceral metastasis was relatively high, and more patients had high expression of $\mathrm{Ki}-67$ and high histological grade (6). Although some progress has been made in the treatment of $\mathrm{MBC}$ in the past decade, according to real-world and clinical trial data, the 5-year survival is still low at $25 \%(6-9)$. The purpose of this retrospective study was to analyze the clinicopathological characteristics of DnMBC and to explore the prognostic factors, in order to provide guidance for clinical diagnosis and treatment.

\section{Patients and methods}

Study population. Data for this study population were obtained from Tianjin Medical University Cancer Hospital. A total of 1,890 patients with advanced breast cancer were treated between January 2008 to December 2017. Patient characteristics are presented in Table I. A total of 171 patients with 
DnMBC were screened out. The criteria for admission were as follows: i) Female patients; ii) primary unilateral breast cancer diagnosed by pathology or imaging with distant metastasis; iii) relatively complete clinical and pathological data, including age of onset, menopause, Ki-67 expression level, hormone receptor status. Human epidermal growth factor receptor 2 (HER2) expression, initial metastasis and treatment and iv) follow-up data are complete. Case exclusion criteria: Male breast cancer, early or locally advanced breast cancer, primary double breast cancer, combined with other malignant tumors, incomplete clinical and pathological data or lost follow-up. In order to adjust for guaranteed time bias, patients only include DnMBC diagnosed early by modern imaging and exclude those who diagnosed shortly after surgery.

Definition. Breast cancer staging is based on the TNM staging guidelines of the seventh edition of the American Joint Committee on Cancer. Estrogen receptor (ER), progesterone receptor (PR) and HER2 were evaluated according to the scoring system recommended by the American Society of Clinical Oncology and the College of American Pathologists (10). Estrogen and progesterone receptor status was assessed using immunohistochemistry (IHC) staining of tissue sections, as previously described $(11,12)$. At least $1 \%$ nuclear positive tumor cells were considered to be positive. ER and/or PR positive were defined as hormone receptor (HR) positive BC. HER2 status by IHC was positive if the score was $3+$ and tumors with indeterminate $(2+)$ IHC scores were considered HER2 positive if amplified by fluorescence in situ hybridization, as previously described $(11,13)$. The rest were classified as negative for analysis. Patients who could not be operated on were clinically staged by clinical palpation and imaging techniques such as B-ultrasound and computed tomography. In this study, patients with single organ metastasis and less than five metastatic lesions were grouped into an oligometastasis group and the rest into a polymetastasis group.

Data analysis. Patient characteristics were tabulated and compared between groups using the chi-square test. Progression-free survival (PFS) was defined as the time from the diagnosis of DnMBC to the progression of the tumor or death. Overall survival (OS) was defined as the time from the diagnosis of DnMBC to death by any cause or the last visit. Survival curves were determined by the Kaplan-Meier method and compared using the log-rank test. Values of 1-year and 3-year PFS; and 3 and 5-year OS were calculated. Multivariate analysis was performed by Cox regression. All P-values reported were two-sided and $\mathrm{P}<0.05$ was considered to indicate a statistically significant difference.

\section{Results}

Baseline patient characteristics. A total of 171 patients with DnMBC were screened out of 1,890 patients with advanced breast cancer treated between January 2008 to December 2017 in the authors' hospital. The baseline characteristics are presented in Table I. Among the patients screened, the median age at diagnosis was 53 years (range, 23-77). Regarding primary tumor size, 14 cases $(8.2 \%)$ were in stage T1, $46(26.9 \%)$ in stage T2, $37(21.6 \%)$ in stage T3 and $64(37.4 \%)$ in stage T4.
Regarding axillary lymph nodes, 10 cases $(5.8 \%)$ were in stage N0, $30(17.5 \%)$ in stage N1, $40(23.4 \%)$ in stage N2 and 81 (47.4\%) in stage N3. A total of $119(69.6 \%)$ were ER positive, $52(30.4 \%)$ were negative; $101(59.1 \%)$ were PR positive and 70 (4\%) were negative. There were 51 cases $(29.8 \%)$ with positive HER 2 and 120 cases $(70.2 \%)$ with negative HER2, 13 cases (7.6\%) with Ki-67<20\%, 117 cases $(68.4 \%)$ with Ki-67 (>20\%) and 77 cases $(45 \%)$ with extensive metastasis and 94 cases (55\%) with oligometastasis, of which 54 cases $(31.6 \%)$ had single bone metastasis, 24 cases (14\%) had lung/pleura metastasis, 15 cases $(8.8 \%)$ had liver metastasis, and $1(0.6 \%)$ case had brain metastasis.

In the present study, almost all of the pathological types are invasive cancers and the pathological diagnosis of the patients mainly came from breast tumor puncture specimens, which may not be able to clearly reveal the pathological details of invasive cancer due to the small amount of tissue contained in the specimens.

Treatments. Of the 171 patients, $150(87.7 \%)$ were treated with chemotherapy initially and $162(94.8 \%)$ were treated with taxane- or anthracycline-based regimens in the first-line treatment, of which 100 cases $(58.5 \%)$ were treated with the combination of anthracyclines and taxanes. In HER2-positive patients, $33(64.7 \%)$ were treated with trastuzumab and 18 (35.3\%) without it. Regarding the operation, 41 patients $(24.0 \%)$ received palliative surgery for the primary tumor, of which 13 patients underwent surgery before the rescue chemotherapy and the remaining 28 patients received surgery after different cycles of chemotherapy. The average time to surgery was 7.3 months (0-22 months) after the diagnosis of DnMBC.

Survival estimates. The median follow-up time of 171 patients was 26 months (2-151 months). The survival rate was calculated using the Kaplan-Meier method. The median PFS was 13 months (10.2-15.8 months), the median OS was 34 months (27.9-40.1 months), the 1-year and 3-year PFS rates were $48.1 \%(48.0-48.2 \%)$ and $10.9 \%(10.85-10.95 \%)$ respectively, and the 3-year and 5-year OS rates were $46.3 \%$ (46.4\%) and $25.7 \%$ (25.6-25.8\%; Fig. 1).

Univariate and multivariate analysis of predictors of survival. Univariate analysis showed that menstrual status, ER status, PR status, HER2 expression, Ki-67 index, molecular typing, initial metastasis and primary breast cancer surgery were associated with PFS ( $\mathrm{P}<0.05$; Table II). Primary tumor size, ER status, PR status, molecular typing, initial metastasis, visceral metastasis, anti-HER2 treatment in HER2 positive patients and primary breast cancer surgery were associated with OS $(\mathrm{P}<0.05$; Table II $)$.

Tables III and VI summarize the results of the multivariable Cox proportional hazards model with hazard ratios $>1.0$ indicating an increased risk of progression or death. ER status and sites of first metastasis (oligometastasis vs. polymetastasis) were highly significant independent predictors of PFS in DnMBC. Women with negative ER status had 1.68 times higher risk of progression compared to women with positive ER status [95\% confidence interval (CI) 1.12-2.53, $\mathrm{P}=0.013$ ], women with polymetastasis had 1.49 times higher risk of progression compared to women with oligometastasis $(95 \%$ 
Table I. Baseline characteristics of patients with de novo metastatic breast cancer.

\begin{tabular}{ll}
\hline Characteristic & Patient, n (total=171) $\%$ \\
\hline
\end{tabular}

Median age, years

(range, years)

Menopausal status

Premenopausal

Postmenopausal

Primary tumor stage

T1

$\mathrm{T} 2$

T3

$\mathrm{T} 4$

Unknown

Regional lymph node stage

N0

N1

N2

N3

Unknown

Estrogen-receptor status

Positive

Negative

Progesterone-receptor status

Positive

Negative

HER2 status

Positive

Negative

Subtype

$\mathrm{HER} 2^{+} / \mathrm{HR}^{ \pm}$

HER2 ${ }^{-} / \mathrm{HR}^{+}$

HER $2 / \mathrm{HR}^{-}$

Ki-67

$<20 \%$

$\geq 20 \%$

Unknown

Visceral metastasis

No

Yes

119

101

120

117

Sites of first metastasis

Oligometastasis

Bone

Lung/pleura

Liver

Brain

Polymetastasis

First-line treatment

Chemotherapy

Surgery

Endocrine

Radiotherapy

Unknown
24

15

77

153

53

Table I. Continued.

\begin{tabular}{lrr}
\hline Characteristic & Patient, $\mathrm{n}($ total=171) & $\%$ \\
\hline Surgery & & \\
Yes & 41 & 24.0 \\
No & 130 & 76.0 \\
First-line chemotherapy & 14 & \\
Anthracycline-containing & 48 & 8.2 \\
Taxane-containing & 100 & 28.1 \\
Anthracycline+Taxanes & 9 & 58.5 \\
Other & & 5.2 \\
Trastuzumab for HER2+ & 33 & 64.7 \\
Yes & 18 & 35.3 \\
No & & \\
Family history of cancer & 22 & 12.9 \\
Yes & 149 & 87.1 \\
No & & \\
\hline
\end{tabular}

14

46

37

64

10

10

30

40

81

10

52

70

51

50

97

24

13

41

95

76

43.3

56.7

8.2

26.9

21.6

37.4

5.8

5.8

17.5

23.4

47.4

5.8

69.6

30.4

59.1

40.9

29.8

70.2

29.2

56.7

14.0

7.6

68.4

24.0

55.6

44.4

55.0

94

31.6

14.0

8.8

0.6

45.0

\section{A}

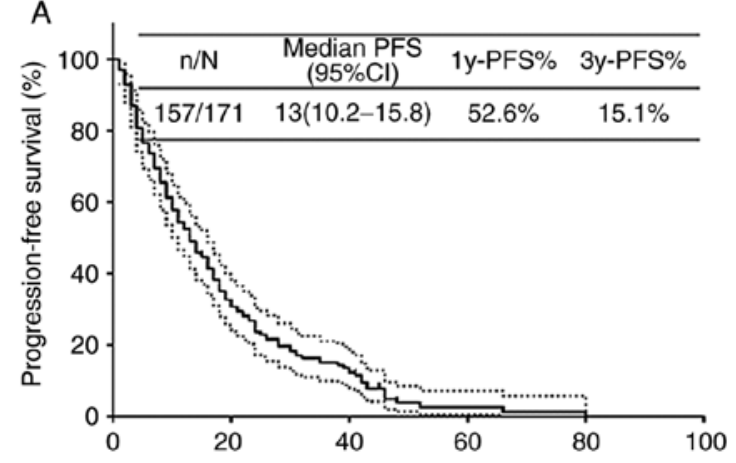

HER2, human epidermal growth factor receptor 2; HR, hormone receptor.
89.5

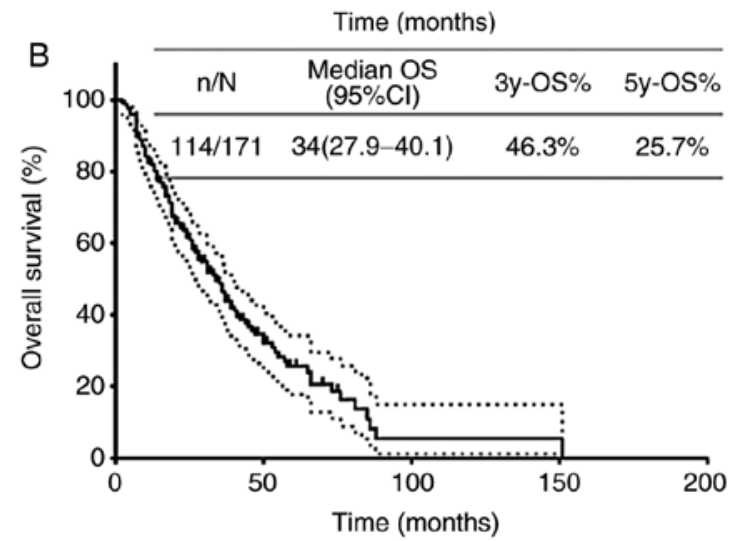

Figure 1. Kaplan-Meier survival curves for PFS and OS of patients with de novo metastatic breast cancer. The Kaplan-Meier (A) PFS and (B) OS curves of patients. PFS, progression free survival; OS, overall survival; CI, confidence interval.

CI 1.00-2.20, $\mathrm{P}=0.048)$. ER status, primary tumor stage and surgical treatment of primary tumors were highly significant independent predictors of OS in DnMBC. Women with negative ER status had 3.11 times higher risk of death compared to women with positive ER status (95\% CI 2.06-4.70; $\mathrm{P}<0.001$ ), 


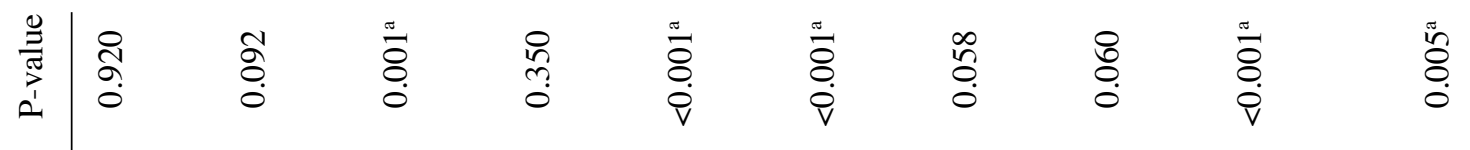

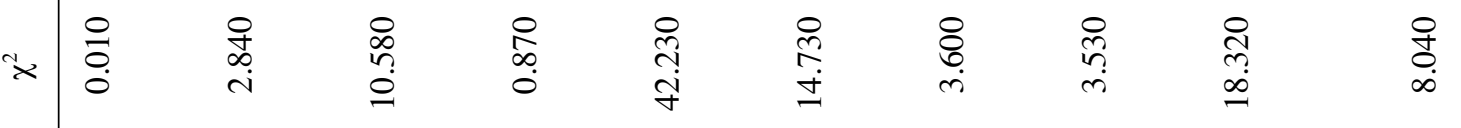

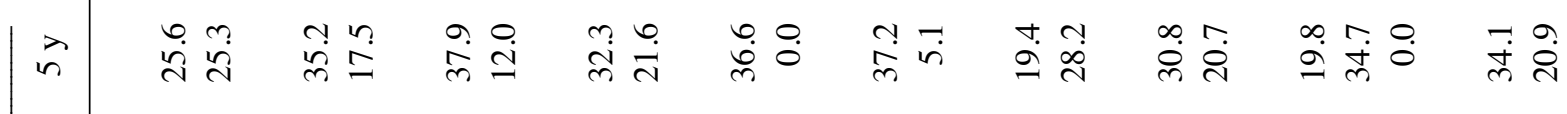

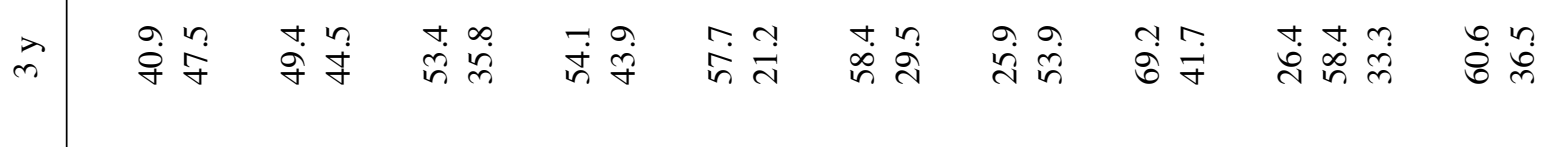

लम

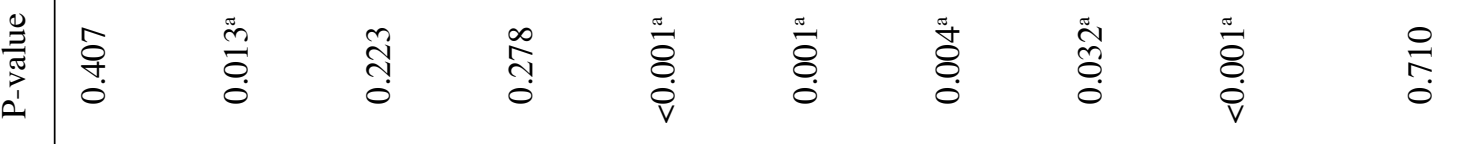

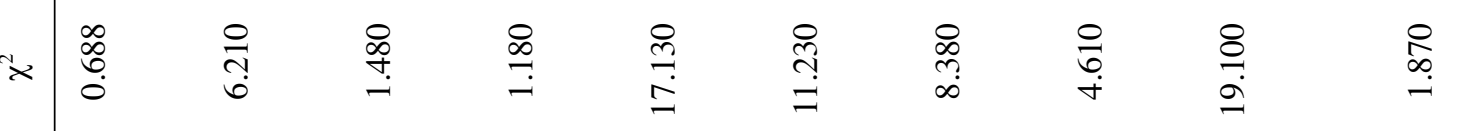

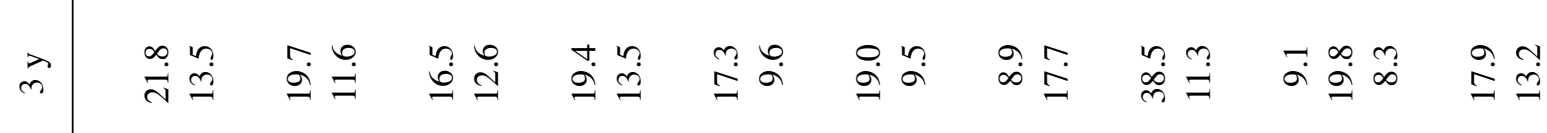

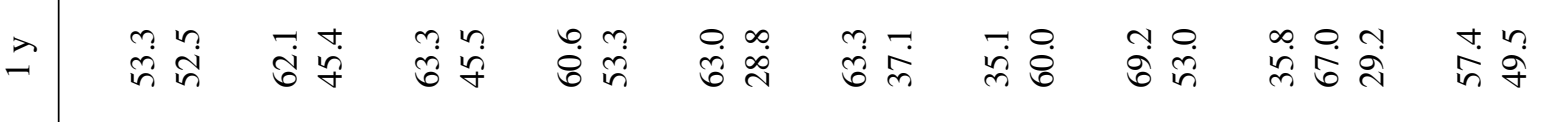

这

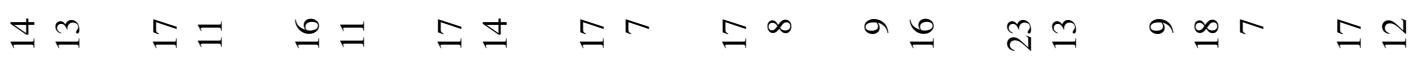

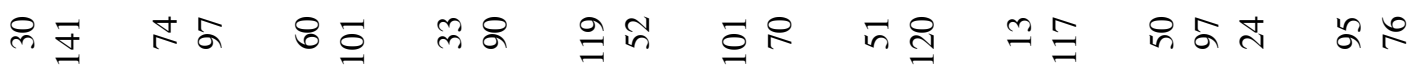

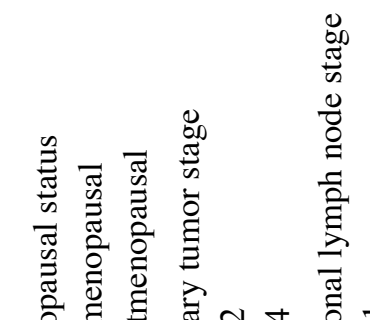

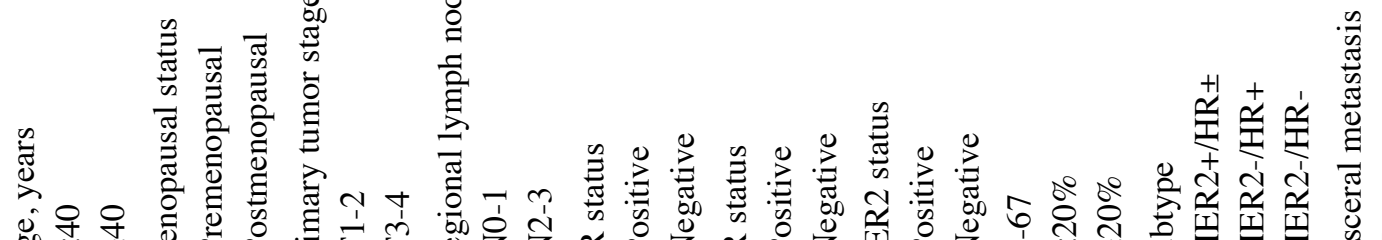

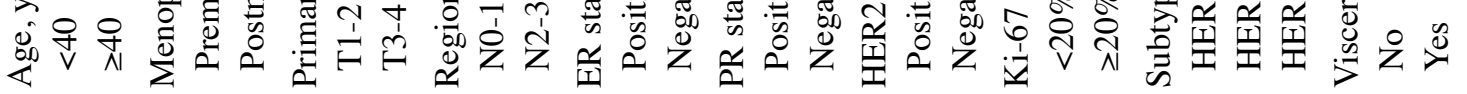




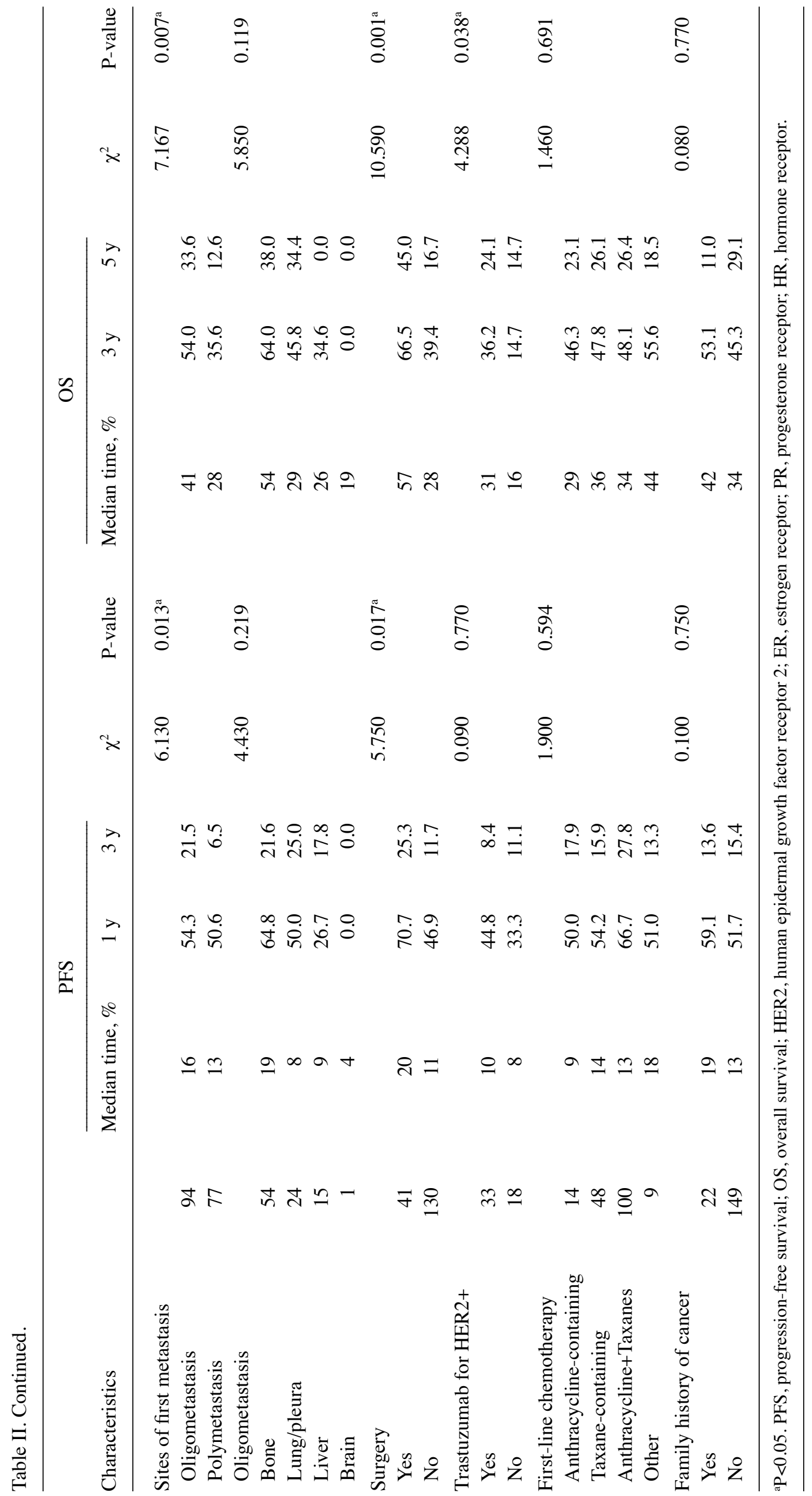


Table III. Multivariate analysis of progression-free survival.

\begin{tabular}{lccc}
\hline Characteristics & HR & $95 \%$ CI & P-value \\
\hline ER status & & & 0.013 \\
$\quad$ ER+ & 1.00 & & \\
ER- & 1.68 & $1.12-2.53$ & 0.048 \\
Sites of first metastasis & & & \\
$\quad$ Oligometastasis & 1.00 & & \\
$\quad$ Polymetastasis & 1.49 & $1.00-2.20$ & \\
\hline
\end{tabular}

HR, hazard ratio; CI, confidence interval; ER, estrogen receptor.

Table IV. Multivariate analysis of overall survival.

\begin{tabular}{lccc}
\hline Characteristics & HR & $95 \%$ CI & P-value \\
\hline ER status & & & $<0.001$ \\
ER+ & 1.00 & & \\
ER- & 3.11 & $2.06-4.70$ & \\
Primary tumor stage & & & 0.003 \\
T1-2 & 1.00 & & \\
T3-4 & 1.88 & $1.24-2.83$ & 0.044 \\
Surgery & & & \\
Yes & 1.00 & & \\
No & 1.70 & $1.02-2.86$ & \\
\hline
\end{tabular}

HR, hazard ratio; CI, confidence interval; ER, estrogen receptor.

women without surgery of the primary tumor had 1.70 times had risk of death compared to women with surgical treatment (95\% CI 1.02-2.86; $\mathrm{P}=0.044)$ and women with tumor stage of T3-4 had 1.88 times higher risk of death compared to women with tumor stage of T1-2 (95\% CI 1.24-2.83, $\mathrm{P}=0.003)$.

Survival analysis of subgroups. Subgroup analysis was performed according to different molecular subtypes, sites of first metastasis and surgical treatment of the DnMBC patients.

Patients were divided into HR+/HER2- (56.7\%), HR $\pm /$ HER 2+ (29.2\%) and HR-/HER2- (14.0\%) groups according to the molecular subtype (Fig. 2). In these groups, the median PFS was 16, 9, 7 months and the 1-year PFS rate was $67.0,35.8$ and $29.2 \%$, respectively $(\mathrm{P}<0.001$; Table II). The median OS was 97, 50, 24 months and the 5-year OS was 34.7, 19.8 and $0.0 \%$, respectively $(\mathrm{P}<0.001$; Table II). PFS and OS in HR+/HER2- group were better than those in the other two groups (Fig. 2B and C), and the difference was statistically significant.

Patients were divided into oligometastasis (55\%) and polymetastasis (45\%) groups according to the sites of first metastasis (Fig. 3A). In these groups, the median PFS was 16 and 13 months, and the 1-year PFS was 54.3 and $50.6 \%$, respectively ( $\mathrm{P}=0.013$; Table $\mathrm{II})$. The median $\mathrm{OS}$ was 41 and 28 months, and the 5-year OS was 33.6 and $12.6 \%$, respectively ( $\mathrm{P}=0.007$; Table II). PFS and OS in the oligometastasis group were better than those in the polymetastasis group (Fig. 3B and C), and the difference was statistically significant.

Patients were divided into the surgery group (24\%) and non-surgery group (76\%) according to the surgical treatment (Fig. 4A). In these groups, the median PFS was 20 and 11 months, and the 1-year PFS was 70.7 and $46.9 \%$, respectively $(\mathrm{P}=0.017$; Table II). The median OS was 57 and 28 months, and the 5-year OS was 45 and $16.7 \%$, respectively $(\mathrm{P}=0.001$; Table II). The PFS and OS in patients with surgery of primary tumor were significantly better than in those without surgery (Fig. 4B and C), and the difference was statistically significant.

Associations between surgery andmolecular subtypes. Patients were divided into $\mathrm{HR}^{+} / \mathrm{HER}^{2}, \mathrm{HR}^{ \pm} / \mathrm{HER}^{+}$and $\mathrm{HR}^{-} / \mathrm{HER} 2-$ groups according to molecular subtype, and were divided into surgery group and non-surgery group according to the surgical treatment. The number of patients who underwent surgical treatment was relatively higher in $\mathrm{HR}^{+} / \mathrm{HER} 2$ - group, but there was no significant difference (Table VI). In the $\mathrm{HR}^{+} / \mathrm{HER} 2-$ group, 27 cases $(15.8 \%)$ were treated with surgery and 70 cases $(40.9 \%)$ without surgery, the OS of the patients who underwent surgical treatment was longer than those without surgery, and the difference was statistically significant. In the $\mathrm{HR}^{ \pm} / \mathrm{HER} 2^{+}$ group, 9 cases $(5.3 \%)$ were treated with surgery and 41 cases (24\%) without surgery, and the OS of the surgery group was longer than that of the non-surgery group, but there was no significant difference. In the HR-/HER2- group, 5 cases $(2.9 \%)$ were treated with surgery and 19 cases $(11.1 \%)$ without surgery, and surgery had no effect on the OS (Fig. 5).

Associations between surgery and first metastasis sites. Patients were divided into oligometastasis and polymetastasis groups according to the sites of first metastasis. The number of patients who underwent surgery was relatively large in the oligometastasis group and the difference was statistically significant (Table V). In the oligometastasis group, 33 cases $(19.3 \%)$ were treated with surgery and 61 cases $(35.7 \%)$ without it, the OS of the patients who received surgical treatment was increased compared with those without it and the difference was statistically significant. In the polymetastasis group, 8 cases $(4.7 \%)$ were treated with surgery with surgery and 69 cases $(40.4 \%)$ without it, and surgery had no effect on OS (Fig. 6).

\section{Discussion}

Cortesi et al (6) made a report based on 119 cases of DnMBC from 2006 to 2009. It was found that their clinicopathological features were more invasive. A total of $\sim 45 \%$ of patients had multiple metastases, $78.1 \%$ had Ki-67 >14\%, $81.9 \%$ with histological grade 3 tumor, $27.5 \%$ were HER2 positive, $59.7 \%$ had initial chemotherapy and nearly $80 \%$ of patients preferred anthracycline or taxane-based chemotherapy regimen. From the analysis of 76 DnMBC cases from 2007 to 2011, Chen et al (14) found that the positive rate of ER, PR and HER2 were 69.7, 56.6, and 27.5\%, respectively. The clinicopathological features and treatment of 171 patients with DnMBC between 2008 and 2017 were analyzed and it was found that the positive rate of HER2 was higher at $29.8 \%$, the percentage of postmenopausal patients and tumor stage 


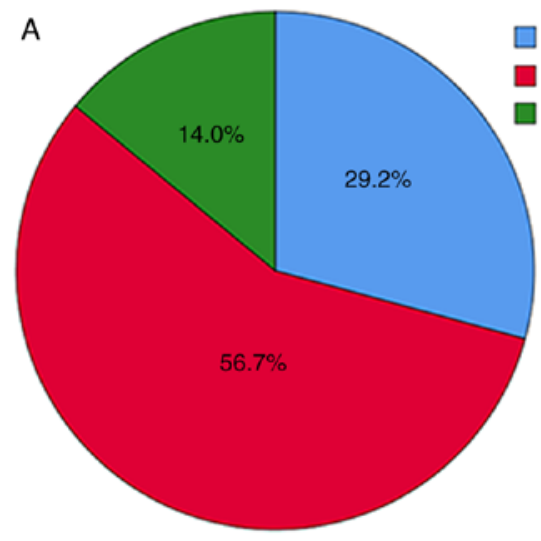

$\square$ GER2+/HR \pm

$\square \mathrm{HER} 2-/ \mathrm{HR}+$

$\square$ HER2-/HR-
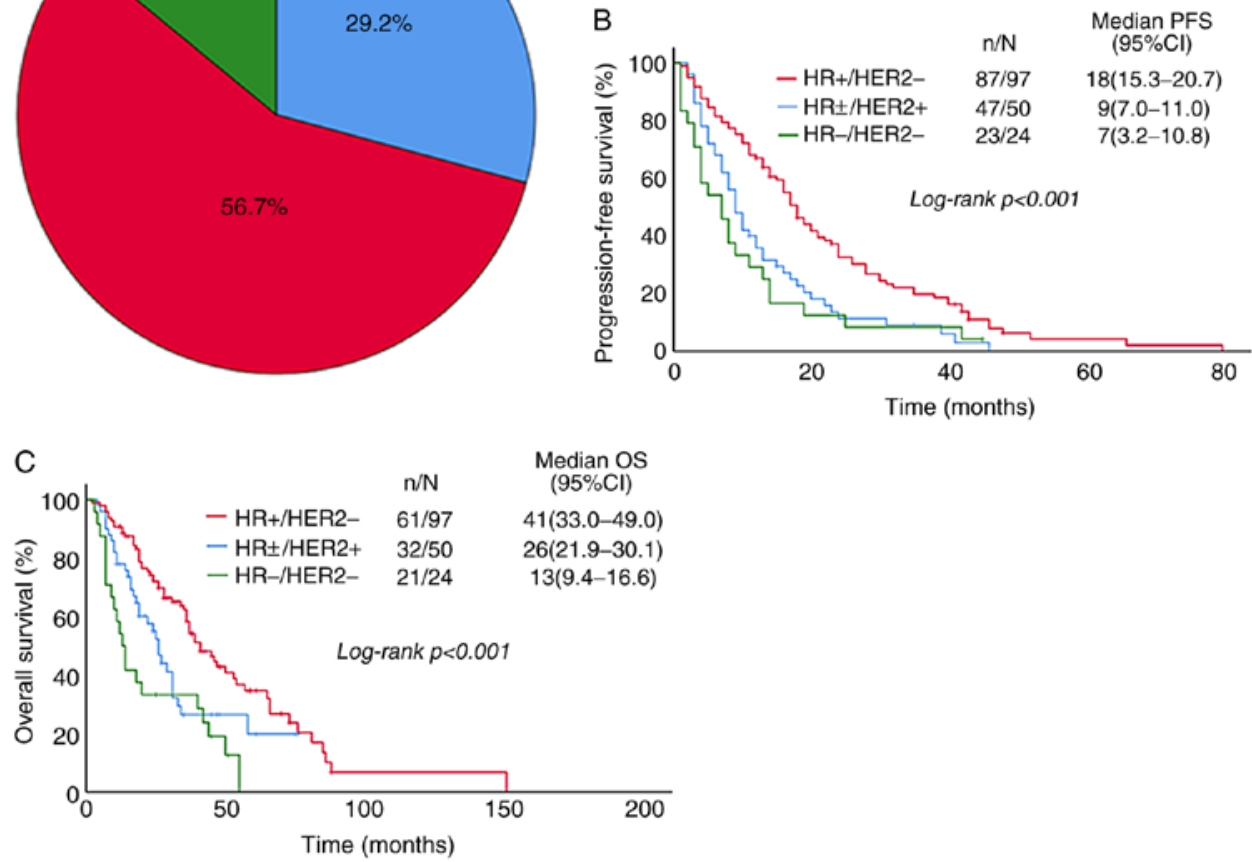

Figure 2. Distribution, and PFS and OS curves of patients with DnMBC, based on different subtypes. (A) The percentage of different subtypes in patients with DnMBC. The Kaplan-Meier (B) PFS and (C) OS curves of patients based on different subtypes. PFS, progression free survival; OS, overall survival; DnMBC, de novo metastatic breast cancer; $\mathrm{CI}$, confidence interval; HR, hormone receptor; HER2, human epidermal growth factor receptor 2; $\mathrm{n} / \mathrm{N}$, number of incidents/total number.
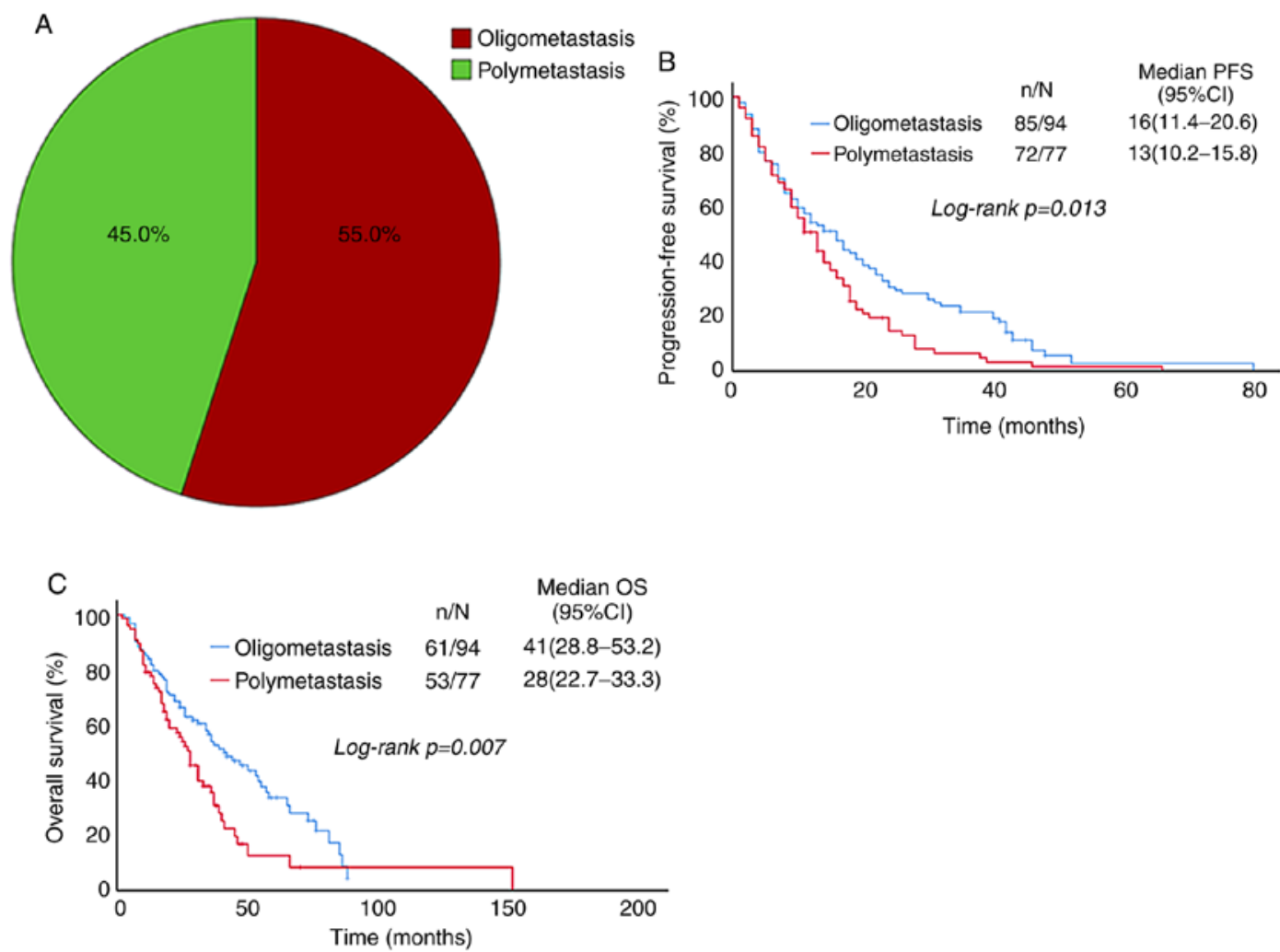

Figure 3. Distribution, and PFS and OS curves of patients with DnMBC, based on different sites of first metastasis. (A) The percentage of different sites of first metastasis in patients with DnMBC. The Kaplan-Meier (B) PFS and (C) OS curves of patients based on different sites of first metastasis. DnMBC, de novo metastatic breast cancer; PFS, progression free survival; OS, overall survival; $\mathrm{CI}$, confidence interval; $\mathrm{n} / \mathrm{N}$, number of incidents/total number. 


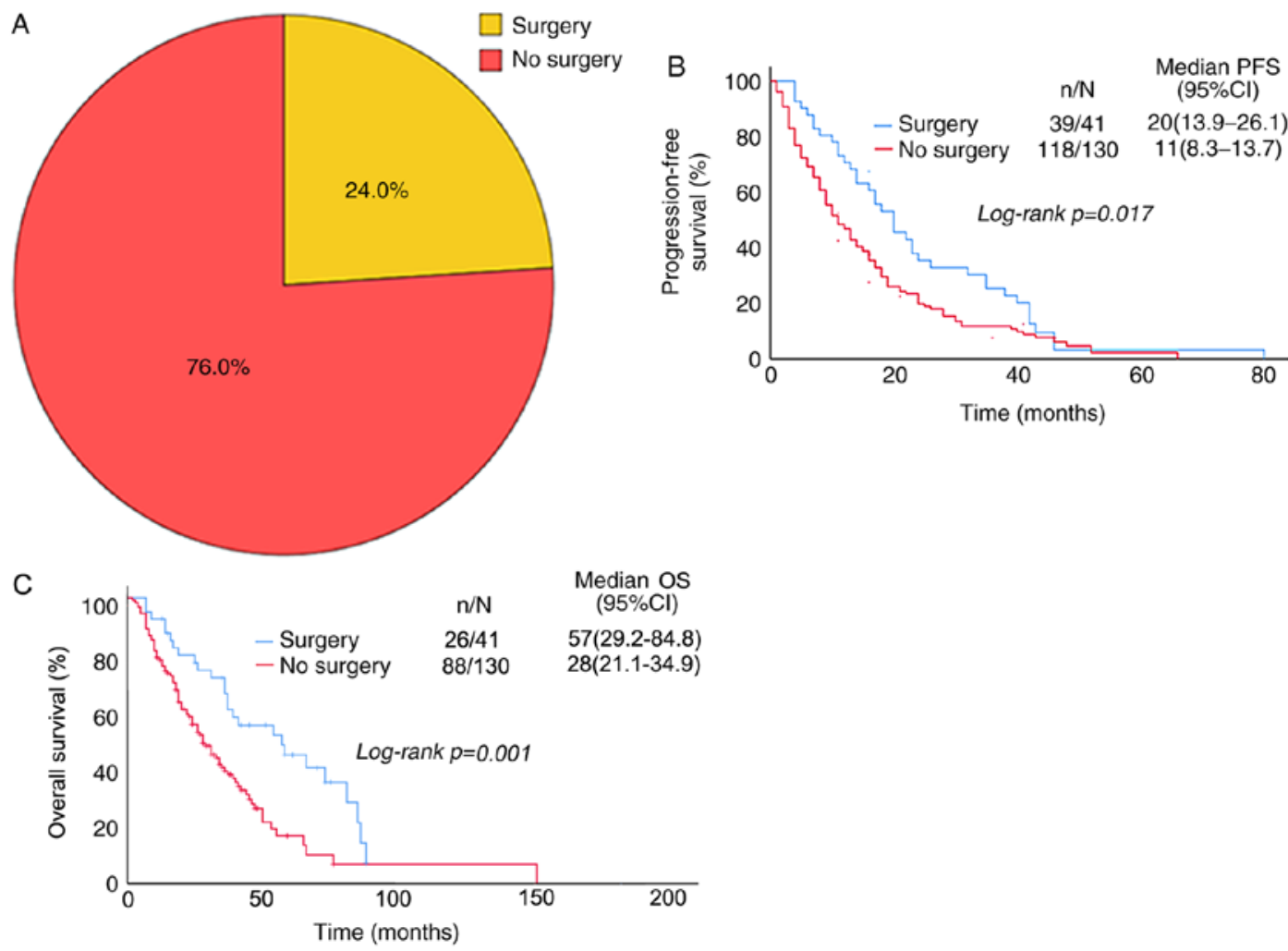

Figure 4. Distribution, and PFS and OS curves of patients with DnMBC, based on surgical treatment for primary tumor. (A) The percentage of surgical treatment for primary tumor in patients with DnMBC. The Kaplan-Meier (B) PFS and (C) OS curves of patients based on surgical treatment for primary tumor. DnMBC, de novo metastatic breast cancer; PFS, progression free survival; OS, overall survival; $\mathrm{CI}$, confidence interval; $\mathrm{n} / \mathrm{N}$, number of incidents/total number.

A
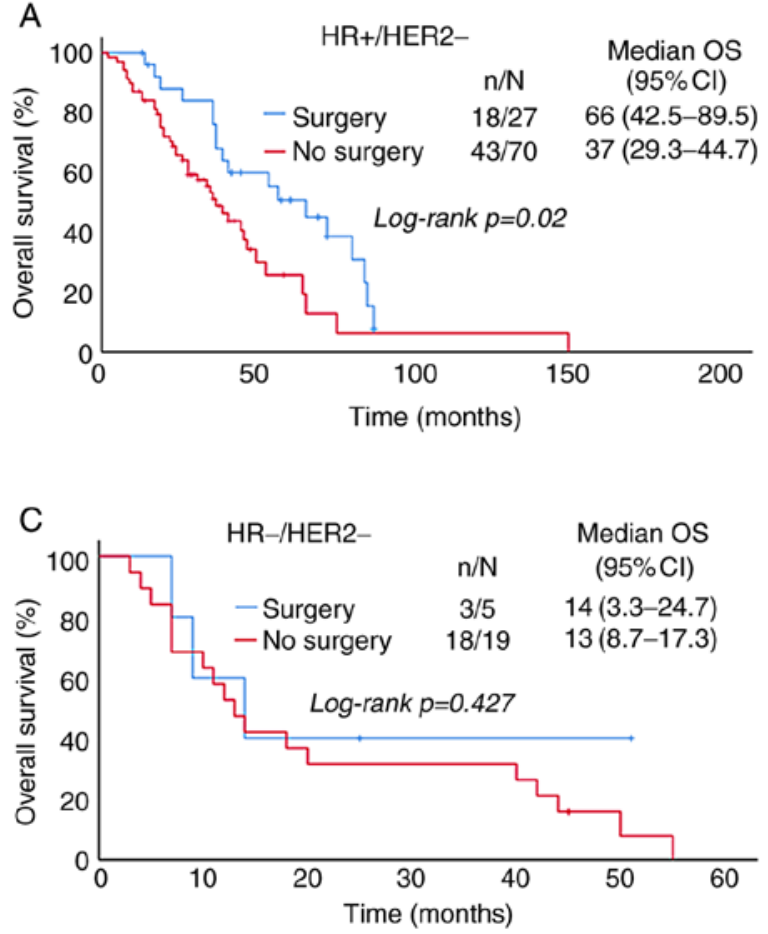

B

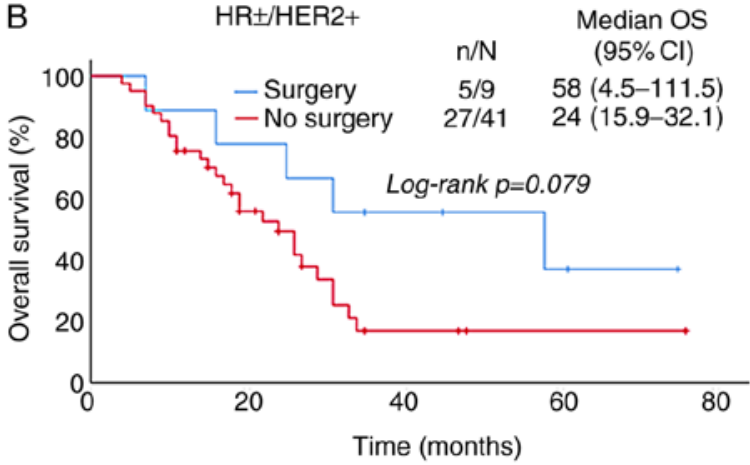

Figure 5. OS curves of patients with de novo metastatic breast cancer, based on different molecular subtypes and surgical treatment. (A) HR+/HER2- subgroup, (B) HR \pm /HER2+ subgroup and (C) HR-/HER2- subgroup. OS, overall survival; CI, confidence interval; HR, hormone receptor; HER2, human epidermal growth factor receptor $2 ; \mathrm{n} / \mathrm{N}$, number of incidents/total number.

T3-4 patients were higher, $68.4 \%$ of patients had Ki- $67 \geq 20 \%$, $45 \%$ had polymetastasis, and $44.4 \%$ with visceral metastasis, which confirmed that DnMBC expresses a more aggressive phenotype. At the same time, the present study found that, 
Table V. Associations between subtypes and surgery of primary tumor surgery.

\begin{tabular}{|c|c|c|c|c|}
\hline Variable & $\begin{array}{c}\text { HR+/HER2- } \\
\text { n (\%) }\end{array}$ & $\begin{array}{c}\mathrm{HR} \pm / \mathrm{HER} 2+ \\
\mathrm{n}(\%)\end{array}$ & $\begin{array}{c}\text { HR-/HER2- } \\
\text { n (\%) }\end{array}$ & P-value \\
\hline Surgery & & & & 0.386 \\
\hline Yes & $27(15.8)$ & $9(5.3)$ & $5(2.9)$ & \\
\hline No & $70(40.9)$ & $41(24.0)$ & $19(11.1)$ & \\
\hline
\end{tabular}

HR, hormone receptor; HER2, human epidermal growth factor receptor 2 .

Table VI. Associations between site of first metastasis and surgery of primary tumor.

\begin{tabular}{lccc}
\hline Variable & $\begin{array}{c}\text { Oligometastasis, } \\
\mathrm{n}(\%)\end{array}$ & $\begin{array}{c}\text { Polymetastasis, } \\
\mathrm{n}(\%)\end{array}$ & P-value \\
\hline Surgery & & & $<0.001$ \\
Yes & $33(19.3)$ & $8(4.7)$ & \\
No & $61(35.7)$ & $69(40.4)$ & \\
\hline
\end{tabular}

similar to studies done by Chen et al (14), the percentage of HR positive in patients with DnMBC was high as well, of which $69.6 \%$ had positive ER status and $59.1 \%$ had positive PR status, which may be associated with better prognosis. Nearly $90 \%$ of the patients in this study received initial chemotherapy and $>90 \%$ of the patients chose taxanes or anthracyclines as the first-line chemotherapy, of whom $58.5 \%$ were treated with the combination of the two drugs. It can be seen that the proportion of chemotherapy is compared with the study by Cortesi et al (6) and there are relatively few studies on the treatment. Considering the differences in traditional concepts among people in different regions, the Chinese are pursuing active and comprehensive treatment, while the Western population is pursuing a higher quality of life, which may have led to the above-mentioned differences in treatment.

Prior reports state that the median survival time of patients with MBC was 2-3 years (7,9,15-18). Andre et al (7) reported that the median survival time of patients with DnMBC was 23 months in 1987-1993, compared with 29 months in 1994-2000, of which most patients had single metastasis especially bone metastasis, $>80 \%$ had received chemotherapy and $51 \%$ had received local surgery or radiation therapy. den Brok et al (18) reported that the median survival time of patients with DnMBC (2001-2009) was 29 months, most of whom were postmenopausal and $>50$ years old. Dawood et al (15) showed that the median survival time of the patients with DnMBC was 39.2 months between 1992 and 2007. Nearly 70\% of the patients in the study were Caucasian and $40 \%$ received surgical treatment for primary tumors, which may be related to the relatively long survival time. In the present study, the median survival time was 34 months and the 5-year OS rate was $25.7 \%$ (DnMBC from 2008 to 2017). According to the above reports, it can be seen that with the continual emergence
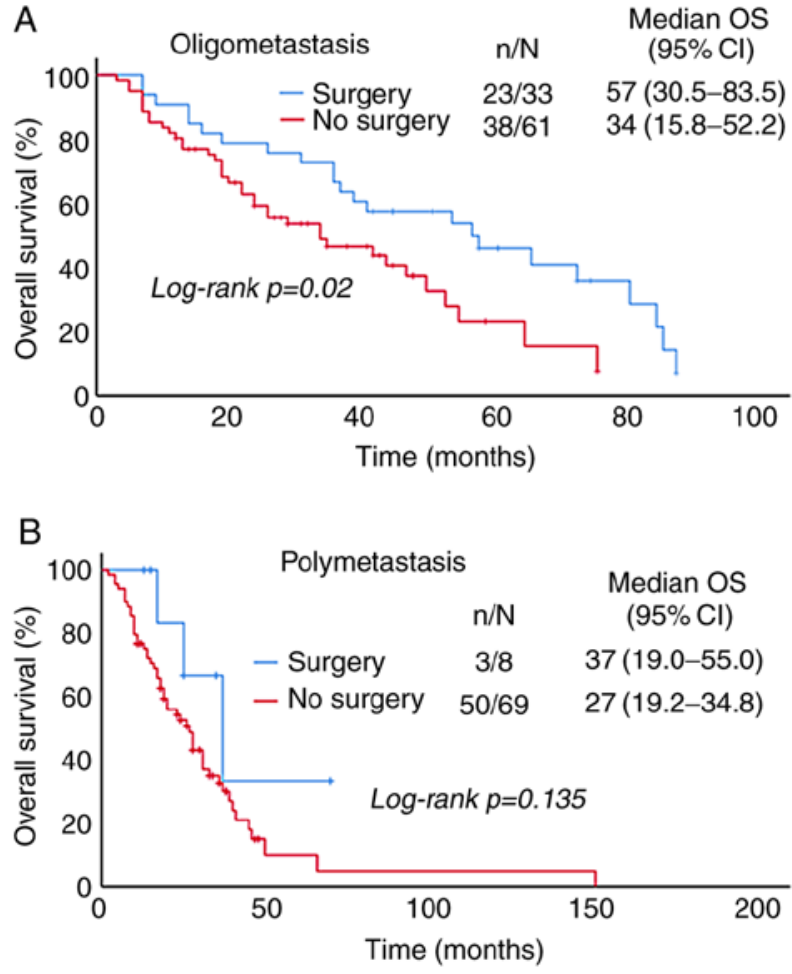

Figure 6. OS curves of patients with de novo metastatic breast cancer, based on site of first metastasis and surgical treatment. (A) Oligometastasis subgroup and (B) Polymetastasis subgroup. OS, overall survival; CI, confidence interval; $\mathrm{n} / \mathrm{N}$, number of incidents/total number.

of new drugs and the developments in treatment in recent years, the median survival time of DnMBC is longer than before. The differences in individual studies may be due to geographical, racial and therapeutic factors.

In this study, univariate analysis showed that poor prognosis was closely related to the following aspects: The primary tumor stage T3-4, ER-negative, PR-negative, polymetastasis, visceral metastasis, patients with positive HER2 status who did not receive anti-HER2 therapy and no palliative surgery for primary tumor. Multivariate analysis showed that ER-negative, primary tumor stage T3-4 and no surgical treatment for primary tumor were independent risk factors for poor prognosis of DnMBC. The results were similar to that of previous studies. Andre et al (7) pointed out that HR negative, multiple metastasis and visceral involvement are risk factors for poor prognosis in DnMBC. Cortesi et al (6) drew the conclusion that patients with negative HR status who did not receive chemotherapy had a poor prognosis.

There are different conclusions on whether surgical treatment of primary tumors will benefit DnMBC. Some studies (19-21) suggested that surgical resection of primary tumors can improve the prognosis of patients with MBC. A retrospective study conducted by Lambertini et al (21) on 113 HER2-positive DnMBC patients showed that PFS and OS were significantly longer in patients who underwent primary tumor surgery, had a lower number of metastatic sites, had minor or no symptoms, received more commonly first-line polychemotherapy treatment, and achieved higher benefit. In the MF07-01 randomized trial (19) with a median follow-up of 40 months, researchers drew a conclusion that primary 
tumor surgery followed by chemotherapy could significantly improve survival times compared with chemotherapy alone in DnMBC and the median OS increased by $\sim 9$ months. This benefit was found especially in patients who were ER positive, HER2 negative, had simple bone metastasis or were younger than 55 . Some studies $(22,23)$ indicated that primary tumor surgery failed to bring survival benefit to MBC patients. A prospective randomized controlled trial (NCT00193778) (22) showed that loco-regional treatment of the primary tumor and axillary nodes had no impact on OS in patients diagnosed with DnMBC, who had responded to frontline chemotherapy. Another prospective trial, TBCRC 013777,(23) also pointed out that among patients who responded to first-line chemotherapy, surgery did not affect OS, irrespective of the tumor subtype. The present data found that local surgical treatment prolonged the PFS and OS, and the 5-year OS rate increased by $28.3 \%$. Furthermore, surgical treatment of the primary tumor was an independent prognostic factor of OS. Considering the selection bias, the proportion of patients with the HR+/HER2-subtype and oligometastasis, especially single bone metastasis, is high in patients who underwent surgical treatment in the current study, so its good prognosis does not exclude this aspect.

Subgroup analysis: The prognosis of patients with primary tumor surgery in different molecular types and sites of first metastasis were analyzed. It was found that patients in the HR+/HER2-group, compared with other subgroups, benefited from surgical treatment and had a longer OS, which was statistically significant. In addition, the effect of surgical treatment was more obvious in the oligometastasis group and the difference was statistically significant as well. Therefore, if patients are in good health and able to undergo surgery, palliative resection of the primary tumor may be considered in DnMBC patients with oligometastasis and the HR+/HER2- subtype. So far, there are no guidelines recommending routine surgical treatment for the primary tumor of DnMBC. Prospective studies with large sample sizes and need to be done to get more clinical data and experience.

Among the molecular subtypes, the post-metastasis OS of MBC had different reports. It has been reported (24) that the longest survival time after metastasis is Lumina subtype. Andre et al (7) speculated that patients with positive HR status had the best prognosis. More reports (7,16,25-30) showed that triple negative breast cancer (TNBC) had the worst prognosis. In the present study, the $\mathrm{HR}^{+} / \mathrm{HER} 2$ subtype had the best prognosis, followed by $\mathrm{HER} 2^{+} / \mathrm{HR}^{ \pm}$ and TNBC had the worst prognosis. The poor prognosis of TNBC may be related to the lack of corresponding hormone therapy and targeted therapy sites.

Hellman et al (31) first proposed the concept of 'oligometastasis' in 1995, as a special state between local recurrence and widespread metastasis. One study (32) suggested that the biological characteristics of the tumor at this stage were relatively stable, the spreading ability was weak and the selectivity for metastatic organs was high. There is no common definition for oligometastasis and the number of metastatic lesions is generally defined as the criterion. A number of studies (33-35) defined the incidence of no more than five metastatic sites as oligometastasis. The present study defined that patients with single organ metastasis and $<5$ metastatic lesions were classified into oligometastasis group, and the rest belonged to the polymetastasis group. Compared with polymetastasis, patients with oligometastasis had better PFS and OS, and the difference was statistically significant. The present study also found that surgical treatment notably improved prognosis in the oligometastasis group, but the benefit was not reflected in the polymetastasis group. It has been pointed out that some patients with oligometastasis can survive for a long time by eliminating oligometastasis through active local treatment such as surgery, radiotherapy and radiofrequency ablation $(34,35)$.

In conclusion, the clinicopathological characteristics of DnMBC are being more invasive and have a higher risk of progression. Palliative surgical treatment may improve the prognosis of $\mathrm{HR}^{+} / \mathrm{HER} 2^{-}$patients with oligometastasis. Therefore, individualized treatment is particularly important. A limitation of the present study was that some subgroups were too small, thus statistical analysis was unable to be performed. Therefore, trials with large samples are needed to verify this conclusion and guide the treatment of DnMBC further.

\section{Acknowledgements}

No applicable.

\section{Funding}

The present study was funded by the National Natural Science Foundation of China (grant no. 81702636), the Anticancer Key Technologies R\&D Program of Tianjin (grant no. 12ZCDZSY16200), the Natural Science Foundation of Tianjin (grant no. 18JCYBJC93500) and the Natural Science Foundation of Tianjin (18JCYBJC91600).

\section{Availability of data and materials}

The datasets used during the present study are available from the corresponding author upon reasonable request.

\section{Authors' contributions}

LZ designed the study, performed the research and wrote the paper. ZL performed the research, analyzed data and wrote the paper. JZ performed research and analyzed data. YW performed research and checked the data. YZ helped performed research and checked the data. ZT designed the study and analyzed data.

\section{Ethics approval and consent to participate}

The present study was approved by the Medical Ethics Committee of Tianjin and written informed consent was obtained from all patients prior to the study start.

\section{Patient consent for publication}

Not applicable.

\section{Competing interests}

The authors declare that they have no competing interests. 


\section{References}

1. Bray F, Ferlay J, Soerjomataram I, Siegel RL, Torre LA and Jemal A: Global cancer statistics 2018: GLOBOCAN estimates of incidence and mortality worldwide for 36 cancers in 185 countries. CA Cancer J Clin 68: 394-424, 2018.

2. Badwe R, Hawaldar R, Nair N, Kaushik R, Parmar V, Siddique S, Budrukkar A, Mittra I and Gupta S: Locoregional treatment versus no treatment of the primary tumour in metastatic breast cancer: An open-label randomised controlled trial. Lancet Oncol 16: 1380-1388, 2015

3. Cardoso F, Spence D, Mertz S, Corneliussen-James D, Sabelko K, Gralow J, Cardoso MJ, Peccatori F, Paonessa D, Benares A, et al: Global analysis of advanced/metastatic breast cancer: Decade report (2005-2015). Breast 39: 131-138, 2018.

4. Dawood S, Broglio K, Gonzalez-Angulo AM, Buzdar AU, Hortobagyi GN and Giordano SH: Trends in survival over the past two decades among white and black patients with newly diagnosed stage IV breast cancer. J Clin Oncol 26: 4891-4898, 2008.

5. Malmgren JA, Mayer M, Atwood MK and Kaplan HG: Differential presentation and survival of de novo and recurrent metastatic breast cancer over time: 1990-2010. Breast Cancer Res Treat 167: 579-590, 2018.

6. Cortesi L, Toss A, Cirilli C, Marcheselli L, Braghiroli B, Sebastiani $\mathrm{F}$ and Federico M: Twenty-years experience with de novo metastatic breast cancer. Int J Cancer 137: 1417-1426, 2015.

7. Andre F, Slimane K, Bachelot T, Dunant A, Namer M, Barrelier A, Kabbaj O, Spano JP, Marsiglia H, Rouzier R, et al: Breast cancer with synchronous metastases: Trends in survival during a 14-year period. J Clin Oncol 22: 3302-3308, 2004.

8. Cortesi L, Cirilli C, Rashid I, Artioli E and Federico M Changes in survival from metastatic breast cancer during the last twenty-years: A population based study in Northern Italy. J Clin Oncol 27: 1125, 2009.

9. Lobbezoo DJ, van Kampen RJ, Voogd AC, Dercksen MW, van den Berkmortel F, Smilde TJ, van de Wouw AJ, Peters FP, van Riel JM, Peters NA, et al: Prognosis of metastatic breast cancer subtypes: The hormone receptor/HER 2-positive subtype is associated with the most favorable outcome. Breast Cancer Res Treat 141: 507-514, 2013.

10. Hammond ME, Hayes DF, Dowsett M, Allred DC, Hagerty KL, Badve S, Fitzgibbons PL, Francis G, Goldstein NS, Hayes M, et al: American society of clinical oncology/college of American pathologists guideline recommendations for immunohistochemical testing of estrogen and progesterone receptors in breast cancer (unabridged version). Arch Pathol Lab Med 134: e48-e72, 2010.

11. Liu F, Lang R, Wei J, Fan Y, Cui L, Gu F, Guo X, Pringle GA, Zhang $\mathrm{X}$ and $\mathrm{Fu} \mathrm{L}$ : Increased expression of SDF-1/CXCR4 is associated with lymph node metastasis of invasive micropapillary carcinoma of the breast. Histopathology 54: 741-750, 2009.

12. Cui LF, Guo XJ, Wei J, Liu FF, Fan Y, Lang RG, Gu F, Zhang XM and Fu L: Overexpression of TNF-alpha and TNFRII in invasive micropapillary carcinoma of the breast: Clinicopathological correlations. Histopathology 53: 381-388, 2008.

13. Qian XL, Wen HY, Yang YL, Gu F, Guo XJ, Liu FF, Zhang L, Zhang XM and Fu L: Assessment of dual-probe Her-2 fluorescent in situ hybridization in breast cancer by the 2013 ASCO/CAP guidelines produces more equivocal results than that by the 2007 ASCO/CAP guidelines. Breast Cancer Res Treat 159: 31-39, 2016.

14. Chen L, Romond E, Chokshi S, Saeed H, Hodskins J, Stevens M, Pasley G, Weiss H and Massarweh S: A prognostic model of early breast cancer relapse after standard adjuvant therapy and comparison with metastatic disease on initial presentation. Breast Cancer Res Treat 136: 565-572, 2012.

15. Dawood S, Broglio K, Ensor J, Hortobagyi GN and Giordano SH: Survival differences among women with de novo stage IV and relapsed breast cancer. Ann Oncol 21: 2169-2174, 2010.

16. Vaz-Luis I, Lin NU, Keating NL, Barry WT, Lii H, Winer EP and Freedman RA: Racial differences in outcomes for patients with metastatic breast cancer by disease subtype. Breast Cancer Res Treat 151: 697-707, 2015 .

17. Lee ES, Jung SY, Kim JY, Kim JJ, Yoo TK, Kim YG, Lee KS, Lee ES, Kim EK, Min JW, et al: Identifying the potential long-term survivors among breast cancer patients with distant metastasis. Ann Oncol 27: 828-833, 2016.

18. den Brok WD, Speers CH, Gondara L, Baxter E, Tyldesley SK and Lohrisch CA: Survival with metastatic breast cancer based on initial presentation, de novo versus relapsed. Breast Cancer Res Treat 161: 549-556, 2017.
19. Soran A, Ozmen V, Ozbas S, Karanlik H, Muslumanoglu M, Igci A, Canturk Z, Utkan Z, Ozaslan C, Evrensel T, et al: A randomized controlled trial evaluating resection of the primary breast tumor in women presenting with de novo stage IV breast cancer: Turkish Study (Protocol MF07-01). J Clin Oncol 34: 1005,2016

20. Thomas A, Khan SA, Chrischilles EA and Schroeder MC: Initial surgery and survival in stage IV breast cancer in the United States, 1988-2011. JAMA Surg 151: 424-431, 2016.

21. Lambertini M, Ferreira AR, Di Meglio A, Poggio F, Puglisi F, Sottotetti F, Montemurro F, Poletto E, Bernardo A, Risi E, et al: Patterns of care and clinical outcomes of HER2-positive metastatic breast cancer patients with newly diagnosed stage IV or recurrent disease undergoing first-line trastuzumab-based therapy: A multicenter retrospective cohort study. Clin Breast Cancer 17: 601-610.e2, 2017.

22. Badwe R, Parmar V, Hawaldar R, Nair N, Kaushik R, Siddique S, Navale A, Budrukkar A, Mittra I and Gupta S: Surgical removal of primary tumor and axillary lymph nodes in women with metastatic breast cancer at first presentation: A randomized controlled trial. Cancer Res 73 (24 Suppl): S2-02, 2013.

23. King TA, Lyman J, Gonen M, Voci A, De Brot M, Boafo C, Sing AP, Hwang ES, Alvarado MD, Liu MC, et al: A prospective analysis of surgery and survival in stage IV breast cancer: TBCRC 013. J Clin Oncol 34: 2359-2365, 2016.

24. Molnár IA, Molnár BÁ, Vízkeleti L, Fekete K, Tamás J, Deák P, Szundi C, Székely B, Moldvay J, Vári-Kakas S, et al: Breast carcinoma subtypes show different patterns of metastatic behavior. Virchows Arch 470: 275-283, 2017.

25. Lobbezoo DJ, van Kampen RJ, Voogd AC, Dercksen MW, van den Berkmortel F, Smilde TJ, van de Wouw AJ, Peters FP, van Riel JM, Peters NA, et al: Prognosis of metastatic breast cancer: Are there differences between patients with de novo and recurrent metastatic breast cancer. Br J Cancer 112: 1445-1451, 2015.

26. Savci-Heijink CD, Halfwerk H, Hooijer GK, Horlings HM, Wesseling $\mathbf{J}$ and van de Vijver MJ: Retrospective analysis of metastatic behaviour of breast cancer subtypes. Breast Cancer Res Treat 150: 547-557, 2015.

27. Tao L, Chu L, Wang LI, Moy L, Brammer M, Song C, Green M, Kurian AW, Gomez SL and Clarke CA: Occurrence and outcome of de novo metastatic breast cancer by subtype in a large, diverse population. Cancer Causes Control 27: 1127-1138, 2016.

28. Vaz-Luis I, Lin NU, Keating NL, Barry WT, Winer EP and Freedman RA: Factors associated with early mortality among patients with de novo metastatic breast cancer: A population-based study. Oncologist 22: 386-393, 2017.

29. Wang H, Zhang C, Zhang J, Kong L, Zhu H and Yu J: The prognosis analysis of different metastasis pattern in patients with different breast cancer subtypes: A SEER based study. Oncotarget 8: 26368-26379, 2017.

30. Wu SG, Li H, Tang LY, Sun JY, Zhang WW, Li FY, Chen YX and He ZY: The effect of distant metastases sites on survival in de novo stage-IV breast cancer: A SEER database analysis. Tumour Biol 39: 1010428317705082, 2017.

31. Hellman S and Weichselbaum RR: Oligometastases. J Clin Oncol 13: 8-10, 1995.

32. Weichselbaum RR and Hellman S: Oligometastases revisited. Nat Rev Clin Oncol 8: 378-382, 2011.

33. Milano MT, Katz AW, Schell MC, Philip A and Okunieff P: Descriptive analysis of oligometastatic lesions treated with curative-intent stereotactic body radiotherapy. Int J Radiat Oncol Biol Phys 72: 1516-1522, 2008.

34. Kobayashi T, Ichiba T, Sakuyama T, Arakawa Y, Nagasaki E, Aiba K, Nogi H, Kawase K, Takeyama H, Toriumi Y, et al: Possible clinical cure of metastatic breast cancer: Lessons from our 30-year experience with oligometastatic breast cancer patients and literature review. Breast Cancer 19: 218-237, 2012.

35. Wong AC, Watson SP, Pitroda SP, Son CH, Das LC, Stack ME, Uppal A, Oshima G, Khodarev NN, Salama JK, et al: Clinical and molecular markers of long-term survival after oligometastasis-directed stereotactic body radiotherapy (SBRT). Cancer 122: 2242-2250, 2016.

This work is licensed under a Creative Commons Attribution-NonCommercial-NoDerivatives 4.0 International (CC BY-NC-ND 4.0) License. 